\title{
Div-Curl Young Measures and Optimal Design in Any Dimension
}

\author{
Pablo PedRegal \\ Departamento de Matemáticas \\ ETSI Industriales \\ Universidad de Castilla-La Mancha \\ 13071 Ciudad Real — Spain \\ pablo.pedregal@uclm.es
}

Received: April 24, 2006

Accepted: October 5, 2006

\begin{abstract}
We explicitly introduce and exploit div-curl Young measures to examine optimal design problems governed by a linear state law in divergence form. The cost is allowed to depend explicitly on the gradient of the state. By means of this family of measures, we can formulate a suitable relaxed version of the problem, and, in a subsequent step, put it in a similar form as the original optimal design problem with an appropriate set of designs and generalized state law. Many of the issues involved has been analyzed elsewhere. The emphasis here is placed on the fact that, by using div-curl Young measures, we make the treatment dimension-independent.
\end{abstract}

Key words: high-dimensional conductivity, cost depending on the field, relaxed formulation.

2000 Mathematics Subject Classification: 49J45, 74P10.

\section{Introduction}

Our motivation is the analysis of a typical optimal design problem in conductivity of the form

$$
\text { Minimize in } \chi: \quad I(\chi)=\int_{\Omega}\left(a_{\alpha} \chi(x)+a_{\beta}(1-\chi(x))|\nabla u(x)|^{2} d x\right.
$$

Supported by project MTM2004-07114 from Ministerio de Educación y Ciencia (Spain) and by project PAI05-029 from JCCM (Castilla-La Mancha). 
subject to

$$
\begin{aligned}
\operatorname{div}[\alpha \chi(x)+\beta(1-\chi(x)) \nabla u(x)] & =0 \quad \text { in } \Omega \\
u & =u_{0} \quad \text { on } \partial \Omega,
\end{aligned}
$$

where $0<\alpha<\beta$ are positive constants, while $a_{\alpha}$ and $a_{\beta}$ could have any sign. The design variable $\chi$ is a characteristic function restricted also by a volume constraint of the type

$$
\int_{\Omega} \chi(x) d x=t_{0}|\Omega|
$$

$\Omega$ is a bounded domain in $\mathbf{R}^{N}, N \geq 2, u_{0} \in H^{1}(\Omega)$, and $t_{0} \in(0,1)$. A more general form can be allowed for the cost density, the state equation, etc, but for the sake of simplicity, we will stick to this simple form of the problem. The most striking feature of this problem is the explicit dependence of the cost density on the gradient of the state $u$.

This kind of problems has been extensively studied because of its importance in applications ([5]). From an analytical perspective, it has been a main motivation to study homogenization of PDE, and tackled by means of these techniques. See [11-13, 20]. The more recent accounts $[1,21]$ are very helpful to gain an overall picture of the problems, the techniques, and the history of homogenization and related concepts and applications. More recently, it has been treated by means of non-convex, vector, variational problems ([17]). We would like to pursue this point of view further.

The approach based on a suitable reformulation of the optimal design problem as a non-convex, vector, variational problem relies on the ability of representing divergence free (solenoidal) vector fields in an appropriate way. This representation depends in an essential way on dimension. For instance, in dimension $N=2$, it is well-known that all such solenoidal fields are of the form $R \nabla u$, rotated gradients with $R$ the $\pi / 2-$, counterclockwise rotation in the plane, while in dimension $N=3$, in most cases divergence-free vector fields $F$ admit the representation $F=\nabla u \times \nabla v$. This decomposition is not unique or canonical, and, what is more important, sometimes does not hold $([14,23])$. The fields $u$ and $v$ are called Clebsch potentials $([7])$. In dimension $N>3$, similar representations can be utilized involving $N-1$ Clebsch potentials which lead to a more complex analysis.

Our main contribution in this note is to develop, in the context of the above optimal design problem, a similar framework as in other previous works ([16]) but without the need to introduce additional potentials. We will rather work directly with divergence-free vector fields and gradients. The associated Young measures ([24]) will be called div-curl Young measures in the spirit of $([6])$. In this way, we can treat the above problem in the same manner, regardless of dimension, and at the same time, simplify some of the computations performed in similar problems in other contexts $([4,8])$.

To explain how the use of div-curl Young measures arise in this kind of optimal design problems, consider a sequence $\chi_{j}$ of feasible designs for the original problem. 
Put

$$
F_{j}=\left[\alpha \chi_{j}(x)+\beta\left(1-\chi_{j}(x)\right)\right] \nabla u_{j}(x),
$$

and consider the sequence of pairs $\left\{\left(F_{j}, \nabla u_{j}\right)\right\}$. This is a sequence of div-curl pairs (div $\left.F_{j}=0, \operatorname{curl} \nabla u_{j}=0\right)$ because of the diffusion state equation. It generates in the limit a div-curl Young measure $\nu=\left\{\nu_{x}\right\}_{x \in \Omega}$. What do we know about the support of this family of measures? It is evident, because of the form of $F_{j}$ coming from the state equation, that

$$
\operatorname{supp}\left(\nu_{x}\right) \subset \Lambda_{\alpha} \cup \Lambda_{\beta}
$$

where

$$
\Lambda_{\gamma}=\left\{(\lambda, \rho) \in \mathbf{R}^{N} \times \mathbf{R}^{N}: \rho=\gamma \lambda\right\}, \quad \gamma=\alpha, \beta .
$$

Therefore we have a div-curl Young measure $\nu$ whose support is restricted to be contained in the union of those two linear manifolds. We claim that this provides a lot of information on $\nu$, which eventually suffices to solve the optimal design problem completely in the sense that we can produce an explicit, relaxed version of it, and whose optimal solutions encode the precise information to build (some) minimizing sequences of designs. Notice that the cost functional can also be written explicitly in terms of $\nu$. If we put

$$
\nu_{x}=t(x) \nu_{x, \alpha}+(1-t(x)) \nu_{x, \beta}
$$

where each $\nu_{x, \gamma}$ has its support contained in $\Lambda_{\gamma}$, then the limit of the costs corresponding to $\chi_{j}$ will be

$$
\int_{\Omega}\left[a_{\alpha} t(x) \int_{\mathbf{R}^{N}}|\lambda|^{2} d \nu_{x, \alpha}^{(1)}(\lambda)+a_{\beta}(1-t(x)) \int_{\mathbf{R}^{N}}|\lambda|^{2} d \nu_{x, \beta}^{(1)}(\lambda)\right] d x .
$$

Here $\nu_{x, \gamma}^{(1)}$ is the projection of $\nu_{x, \gamma}$ onto the first copy of $\mathbf{R}^{N}$.

To state our main theorem, we introduce a bit of notation. For $0<\alpha<\beta, a_{\alpha}$, $a_{\beta}$ as before, let $m(t, \lambda, \rho)$ stand for the minimum value of the linear programming problem

$$
\text { Minimize in }\left(s_{\alpha}, s_{\beta}\right) \in \mathbf{R}^{2}: \quad a_{\alpha} t s_{\alpha}+a_{\beta}(1-t) s_{\beta}
$$

subject to the constraints

$$
\begin{gathered}
\lambda \cdot \rho=t \alpha s_{\alpha}+(1-t) \beta s_{\beta}, \\
s_{\alpha} \geq \frac{1}{t^{2}(\beta-\alpha)^{2}}|\beta \lambda-\rho|^{2}, \quad s_{\beta} \geq \frac{1}{(1-t)^{2}(\beta-\alpha)^{2}}|\rho-\alpha \lambda|^{2} .
\end{gathered}
$$

Further if

$$
\rho=\frac{2 \alpha \beta+t(1-t)(\beta-\alpha)^{2}}{2[\alpha(1-t)+\beta t]} \lambda+\frac{t(1-t)(\beta-\alpha)^{2}}{2[\alpha(1-t)+\beta t]}|\lambda| s, \quad s \in \mathbf{R}^{N}, \quad|s| \leq 1,
$$

set

$$
\psi(t, s, \lambda)=m(t, \rho, \lambda)
$$


and

$$
\varphi(t, s, \lambda)=\frac{2 \alpha \beta+t(1-t)(\beta-\alpha)^{2}}{2[\alpha(1-t)+\beta t]} \lambda+\frac{t(1-t)(\beta-\alpha)^{2}}{2[\alpha(1-t)+\beta t]}|\lambda| s .
$$

Theorem 0.1. Consider the problem

$$
\text { Minimize in }(t, s, u): \quad \int_{\Omega} \psi(t(x), s(x), \nabla u(x)) d x
$$

subject to

$$
\begin{gathered}
0 \leq t(x) \leq 1, \quad \int_{\Omega} t(x) d x=t_{0}|\Omega|, \quad s(x) \in \mathbf{R}^{N},|s(x)| \leq 1, \\
\operatorname{div} \varphi(t(x), s(x), \nabla u(x))=0 \text { in } \Omega, \quad u=u_{0} \text { on } \partial \Omega .
\end{gathered}
$$

This problem is a relaxation of the original optimal design problem in the sense:

- the infimum for the original problem equals the minimum for this problem;

- optimal solutions for this problem encode in a precise way (see below) the optimal microestructures for the original problem.

Notice that by taking $t=0$ and $t=1$, the relaxed problem reduces to the original one. We will specify later (formulae in Lemma 3.1 of section 3) this "precise way" in which optimal microestructures can be built from optimal solutions of the relaxed version. This is always done through div-curl laminates of at most second-order (see section 3 and [16]).

We know, because it is a relaxation, that this is a well-posed optimal design problem whose optimal solutions can be approximated by exploiting optimality conditions, or by looking for descent directions. We will pursue this approach in a forthcoming work. The explicit form for $\psi$ can be given for each choice of the coefficients $a_{\alpha}$ and $a_{\beta}$. We include a particular example at the end of section 3 .

Let us emphasize again that the main contribution of this note is to free the analysis of previous works (see references above), which is only valid for the case of 2 or 3 dimensions, to any dimension without the need of making any distinction based on the dimension of the problem. We will not therefore prove again formal results and computations which are exactly the same in our context. In particular, we simply incorporate facts which have been discussed in detail in $[2,16,17]$ with some indications when appropriate. We refer readers to these papers for a full discussion.

\section{Div-Curl Young measures}

This is the class of Young measures associated with a sequence of pairs of vector fields $\left\{\left(F_{j}, G_{j}\right)\right\}$ such that

$$
F_{j}: \Omega \rightarrow \mathbf{M}^{m \times N}, \quad G_{j}: \Omega \rightarrow \mathbf{M}^{m \times N}
$$


are bounded sequences in $L^{2}(\Omega)$, where $\Omega \subset \mathbf{R}^{N}$ is a regular, bounded domain, and

$$
\operatorname{div} F_{j}=0, \quad \operatorname{curl} G_{j}=0 \quad \text { in } \Omega,
$$

in a weak sense. Under the additional assumption of simple-connectedness of $\Omega$, we can put $G_{j}=\nabla u_{j}$ for a certain $u_{j} \in H^{1}\left(\Omega ; \mathbf{R}^{n}\right)$, so that we will consider from the start sequences of pairs $\left\{\left(F_{j}, \nabla u_{j}\right)\right\}$ and forget about the simple-connectedness of $\Omega$. In what follows, more general assumptions can be allowed on the spaces of fields in the sense that the divergence-free and curl-free requirements for $F_{j}$ and $G_{j}$, respectively, can be relaxed.

We know $([3,9,15,22])$ that we can always associate with such a sequence (rather a subsequence) of pairs a family of probability measures, its Young measure $\nu=$ $\left\{\nu_{x}\right\}_{x \in \Omega}$, supported in $\mathbf{M}^{m \times N} \times \mathbf{M}^{m \times N}$, such that whenever the sequence of functions $\left\{\phi\left(x, F_{j}(x), \nabla u_{j}(x)\right)\right\}$ weakly converges in $L^{1}(\Omega)$ for some Carathéodory integrand $\phi$, the weak limit is given by

$$
\bar{\phi}(x)=\int_{\mathbf{M}^{m \times N} \times \mathbf{M}^{m \times N}} \phi(x, \rho, \lambda) d \nu_{x}(\rho, \lambda) .
$$

More formally, we adopt the following definition.

Definition 1.1. A family of probability measures $\nu=\left\{\nu_{x}\right\}_{x \in \Omega}$ is called a $\left(L^{2}-\right)$ div-curl Young measure if there exists a sequence of pairs of vector fields $F_{j}$ in $L^{2}\left(\Omega ; \mathbf{M}^{m \times N}\right)$, and $u_{j}$ in $H^{1}\left(\Omega ; \mathbf{R}^{m}\right)$, such that

$$
\operatorname{div} F_{j} \rightarrow 0 \text { in } H^{-1}\left(\Omega ; \mathbf{R}^{m}\right), \quad\left\{\left|F_{j}\right|^{2}\right\},\left\{\left|\nabla u_{j}\right|^{2}\right\} \text { are equiintegrable in } \Omega,
$$

and the Young measure associated with $\left\{\left(F_{j}, \nabla u_{j}\right)\right\}$ is $\nu$.

The whole point is to understand better this class of measures, and, in particular, how the property of being a pair with an essentially-divergence-free component and a gradient, translates into the structure of the Young measure itself. We are specially interested, bearing in mind the application to optimal design problems, in exploring the interaction between the divergence-free and the curl-free components of the sequence of pairs. It is important to point out that this is a very particular situation of the rather general framework of $A$-quasiconvexity, and $A$-Young measures as introduced and discussed in [6]. See also [18]. We will in fact rely on this work for all the proofs and rigorous facts of this section. Indeed, the rest of this section is a reminder of the main facts about this class of measures whose proofs can be found in that reference.

The main constraint on this class of measures, from our point of view, is, however, an immediate consequence of the classic and well-known div-curl lemma $([10,19,21])$.

Lemma 1.2. Let $\left\{F_{j}\right\}$ be a sequence of bounded fields in $L^{2}\left(\Omega ; \mathbf{M}^{m \times N}\right)$ converging weakly to $F$, such that $\left\{\operatorname{div} F_{j}\right\}$ is bounded in $L^{2}\left(\Omega ; \mathbf{R}^{m}\right)$, and let $\left\{\nabla u_{j}\right\}$ be a bounded sequence of gradients in $H^{1}\left(\Omega ; \mathbf{R}^{m}\right)$ converging weakly to $\nabla u$. Then

$$
F_{j}\left(\nabla u_{j}\right)^{T} \rightarrow F \nabla u^{T}
$$


in the sense of distributions.

By a direct application of this fundamental result to div-curl Young measures, we obtain the fundamental commutation property.

Lemma 1.3. If $\nu=\left\{\nu_{x}\right\}_{x \in \Omega}$ is a div-curl Young measure, then for a.e. $x \in \Omega$,

$$
\int_{\mathbf{M}^{m \times N} \times \mathbf{M}^{m \times N}} \rho \lambda^{T} d \nu_{x}(\rho, \lambda)=\int_{\mathbf{M}^{m \times N}} \rho d \nu_{x}^{(1)}(\rho) \int_{\mathbf{M}^{m \times N}} \lambda^{T} d \nu_{x}^{(2)}(\lambda),
$$

where $\nu_{x}^{(i)}, i=1,2$, are the marginals on the two components, respectively.

Notice that the product $\rho \lambda^{T}$ is an $m \times m$ matrix.

The localization principle is also valid for this class of Young measures. This basically says that if $\nu=\left\{\nu_{x}\right\}_{x \in \Omega}$ is a div-curl Young measure, then for a.e. $a \in \Omega$ each individual member $\nu_{a}$ is in its own right a homogeneous (not dependent on $x \in \Omega$ ), div-curl Young measure, which means that it can be generated by a new sequence of pairs $\left\{\left(F_{j}^{a}, \nabla u_{j}^{a}\right)\right\}$ (depending on $a$ ) with $\operatorname{div}\left(F_{j}^{a}\right) \rightarrow 0$ in $\Omega$. These pairs of fields are obtained through a typical process of localization or blow-up around $a$ from the pairs determining the initial div-curl Young measure. This is standard $([6,15])$. Conversely, we can glue together specific, homogeneous div-curl Young measures, one for each point $x \in \Omega$, in a big div-curl Young measure all over $\Omega$. The only requirement is that the resulting barycenter be consistent with the div-curl constraint.

Lemma $1.4([6])$. A family of probability measures $\nu=\left\{\nu_{x}\right\}_{x \in \Omega}$ is a div-curl Young measures if and only if:

- For a.e. $x \in \Omega$, each individual $\nu_{x}$ is a homogeneous, div-curl Young measure itself.

- There exists a divergence-free vector field $F$ in $L^{2}\left(\Omega ; \mathbf{R}^{m}\right)$, and a field $u \in$ $H^{1}\left(\Omega ; \mathbf{R}^{m}\right)$ such that

$$
F(x)=\int_{\mathbf{M}^{m \times N}} \rho d \nu_{x}^{(1)}(\rho), \quad \nabla u(x)=\int_{\mathbf{M}^{m \times N}} \lambda d \nu_{x}^{(2)}(\lambda) .
$$

There is another important issue that refers to a specific, general way of constructing explicitly div-curl Young measures. This is the analogue of laminates for gradient Young measures ([15]), and it is based on the same principle. This is again standard. The basic construction and the typical recursive procedure are recorded in the next lemma.

Lemma 1.5. Suppose that $\rho_{i}, \lambda_{i}, i=1,2$ are four $m \times N$ matrices such that

$$
\left(\rho_{2}-\rho_{1}\right)\left(\lambda_{2}^{T}-\lambda_{1}^{T}\right)=0
$$


as $m \times m$ matrices. Then the probability measure

$$
\mu=t \delta_{\left(\rho_{1}, \lambda_{1}\right)}+(1-t) \delta_{\left(\rho_{2}, \lambda_{2}\right)}
$$

is a div-curl Young measure for all $t \in[0,1]$.

If $\nu_{1}$ and $\nu_{2}$ are two div-curl Young measures with barycenters $\left(\rho_{1}, \lambda_{1}\right)$ and $\left(\rho_{2}, \lambda_{2}\right)$, respectively, such that (3) holds, then

$$
\mu=t \nu_{1}+(1-t) \nu_{2}
$$

is a div-curl Young measure too for any $t \in[0,1]$.

\section{The variational reformulation}

We start this section by stating the genuine variational reformulation of the original optimal design problem. To that end, consider the integrands defined by

$$
W(\rho, \lambda)=\left\{\begin{array}{ll}
a_{\alpha}|\lambda|^{2}, & \text { if } \rho=\alpha \lambda, \\
a_{\beta}|\lambda|^{2}, & \text { if } \rho=\beta \lambda, \\
+\infty, & \text { else, }
\end{array} \quad V(\rho, \lambda)= \begin{cases}1, & \text { if } \rho=\alpha \lambda, \\
0, & \text { if } \rho=\beta \lambda, \\
+\infty, & \text { else. }\end{cases}\right.
$$

Then it is an elementary exercise to see that the initial problem can be reformulated in the following terms

$$
\text { Minimize in }(F, u): \quad \int_{\Omega} W(F(x), \nabla u(x)) d x
$$

subject to

$$
F \in L^{2}\left(\Omega ; \mathbf{M}^{m \times N}\right), \quad u \in H^{1}\left(\Omega ; \mathbf{R}^{m}\right),
$$

$\operatorname{div} F=0$ weakly in $\Omega, \quad u=u_{0}$ on $\partial \Omega, \quad \int_{\Omega} V(F(x), \nabla u(x)) d x=t_{0}|\Omega|$.

This equivalent formulation suffers from the same troubles as the initial problem, so that it is in need of relaxation. Because this variational problem has been formulated in a local fashion, its relaxation can be examined by means of Young measures as is typically done for variational problems $([3,9,15,22])$. In this way, we are led to understand Young measures corresponding to sequences of pairs $\left\{\left(F_{j}, \nabla u_{j}\right)\right\}$ with $\operatorname{div} F_{j}=0$ in $\Omega$. These are div-curl Young measures. If we expect to gain something by looking at the relaxed formulation in terms of this family of measures, it is necessary to know them better. We have already stated many of their general properties in the preceding section. We now specialized some of those properties to the situation of our design problem.

Suppose that $\left\{\chi_{j}\right\}$ is a sequence of admissible characteristic functions for our initial optimal design problem, so that if the sequence of fields $F_{j}$ is given by (1), then the 
Young measure $\nu=\left\{\nu_{x}\right\}_{x \in \Omega}$ associated with the sequence of pairs $\left\{\left(F_{j}, \nabla u_{j}\right)\right\}$ is a div-curl Young measure as defined in the previous section. Because of the specific relationship between $F_{j}$ and $\nabla u_{j}$, it is also elementary to conclude as in (2) for a.e. $x \in \Omega$. In this way, each individual $\nu_{x}$ is a homogeneous div-curl Young measure supported in the union of those two linear manifolds. The main contribution in this section is the converse of this statement.

Proposition 2.1. A family of probability measures $\nu=\left\{\nu_{x}\right\}_{x \in \Omega}$ corresponds to a sequence of pairs

$$
\left\{\left(\left[\alpha \chi_{j}(x)+\beta\left(1-\chi_{j}(x)\right)\right] \nabla u_{j}(x), \nabla u_{j}(x)\right)\right\}
$$

where $\chi_{j}$ is a sequence of feasible characteristic functions for our original optimal design problem, if and only if:

- Each $\nu_{x}$ as such is a homogeneous div-curl Young measure supported in the union $\Lambda_{\alpha} \cup \Lambda_{\beta}$, and there is $t: \Omega \rightarrow[0,1]$ such that

$$
\int_{\Omega} t(x) d x=t_{0}|\Omega|, \quad \nu_{x}=t(x) \nu_{x, \alpha}+(1-t(x)) \nu_{x, \beta}
$$

with each $\nu_{x, \gamma}$ supported in $\Lambda_{\gamma}$.

- There exists a divergence-free vector field $F$ in $L^{2}\left(\Omega ; \mathbf{R}^{m}\right)$, and a field $u \in$ $H^{1}\left(\Omega ; \mathbf{R}^{m}\right)$ such that

$$
F(x)=\int_{\mathbf{M}^{m \times N}} \rho d \nu_{x}^{(1)}(\rho), \quad \nabla u(x)=\int_{\mathbf{M}^{m \times N}} \lambda d \nu_{x}^{(2)}(\lambda) .
$$

Proof. The proof amounts to showing that if we have a family $\nu=\left\{\nu_{x}\right\}_{x \in \Omega}$ of probability measures verifying the conditions on the statement, then there is a sequence of feasible characteristic functions $\left\{\chi_{j}\right\}$ so that $\nu=\left\{\nu_{x}\right\}_{x \in \Omega}$ is precisely the div-curl Young measure associated with the pairs in (4). Notice that this is essentially what Lemma 1.4 says. What is at stake is the fact that the sequence of generating pairs in (4) should take on values on the two manifolds everywhere, and not only "approximately." This sort of arguments were used in a careful way in [2], but we include here the main idea of the proof in the context of div-curl Young measures.

Assume, then, that we have a family of probability measures $\nu=\left\{\nu_{x}\right\}_{x \in \Omega}$ such that the conditions on the statement hold. By Lemma 1.4, there exists a sequence of pairs $\left\{\left(F_{j}, \nabla u_{j}\right)\right\}$ such that theirs squares are equiintegrable in $\Omega$, div $F_{j} \rightarrow 0$ in $H^{-1}\left(\Omega ; \mathbf{R}^{m}\right)$, and its corresponding Young measure is precisely the family $\nu$. This implies that for a.e. $x \in \Omega$, and for $j$ large, the pairs $\left\{\left(F_{j}, \nabla u_{j}\right)\right\}$ have to be close to one of the two manifolds $\Lambda_{\alpha}$ or $\Lambda_{\beta}$. More precisely, put

$$
\phi_{j}(x)=\min \left\{\left|F_{j}(x)-\alpha \nabla u_{j}(x)\right|^{2},\left|F_{j}(x)-\beta \nabla u_{j}(x)\right|^{2}\right\} .
$$


This function measures the distance from the pair $\left(F_{j}, \nabla u_{j}\right)$ to the union of the two manifolds. It is clear that there is a characteristic function $\chi_{j}(x)$ such that

$$
\phi_{j}(x)=\left|F_{j}(x)-\left(\chi_{j}(x) \alpha+\left(1-\chi_{j}(x)\right) \beta\right) \nabla u_{j}(x)\right|^{2}
$$

and

$$
\int_{\Omega} \chi_{j}(x) d x \rightarrow t_{0}|\Omega|
$$

Because of the integrability of $\left\{\left|\nabla u_{j}\right|^{2}\right\}$, we can modify a bit this characteristic function, in a small subset of negligible measure as $j \rightarrow \infty$, so that

$$
\int_{\Omega} \chi_{j}(x) d x=t_{0}|\Omega|
$$

and still $\left\{\phi_{j}\right\}$ is equiintegrable in $\Omega$. For this sequence of functions the representation in terms of the Young measure $\nu$ holds (because of the integrability just mentioned) and

$$
\lim _{j \rightarrow \infty} \int_{\Omega} \phi_{j}(x) d x=\int_{\Omega} \int_{\mathbf{R}^{N} \times \mathbf{R}^{N}} \min \left\{|\rho-\alpha \lambda|^{2},|\rho-\beta \lambda|^{2}\right\} d \nu_{x}(\rho, \lambda) d x=0,
$$

because each $\nu_{x}$ is supported in the union of the two manifolds where one of the two terms in the minimum vanishes while the other is positive.

Let $\mu=\left\{\mu_{x}\right\}_{x \in \Omega}$ be the Young measure associated with the sequence of pairs

$$
\left\{\left(\left(\chi_{j} \alpha+\left(1-\chi_{j}\right) \beta\right) \nabla v_{j}, \nabla v_{j}\right)\right\}, \quad H_{j}=\left(\chi_{j} \alpha+\left(1-\chi_{j}\right) \beta\right) \nabla v_{j},
$$

where $v_{j}$ are the solutions of

$$
\operatorname{div}\left[\left(\chi_{j} \alpha+\left(1-\chi_{j}\right) \beta\right) \nabla v_{j}\right]=0 \text { in } \Omega, \quad v_{j}=u_{0} \text { on } \partial \Omega .
$$

We claim that $v_{j}-u_{j}$ converges strongly to zero in $H^{1}\left(\Omega ; \mathbf{R}^{m}\right)$. Indeed, if we put $\gamma_{j}=\chi_{j} \alpha+\left(1-\chi_{j}\right) \beta$, we have

$$
\int_{\Omega} \alpha\left|\nabla u_{j}(x)-\nabla v_{j}(x)\right|^{2} d x \leq \int_{\Omega}\left\langle\gamma_{j}(x)\left(\nabla u_{j}(x)-\nabla v_{j}(x)\right), \nabla u_{j}(x)-\nabla v_{j}(x)\right\rangle d x .
$$

The right-hand side can be rewritten as

$$
\int_{\Omega}\left\langle\gamma_{j} \nabla u_{j}-F_{j}, \nabla u_{j}(x)-\nabla v_{j}(x)\right\rangle+\left\langle F_{j}(x)-H_{j}(x), \nabla u_{j}(x)-\nabla v_{j}(x)\right\rangle d x .
$$

Because $\operatorname{div} F_{j} \rightarrow 0$, div $H_{j}=0$, and $u_{j}-v_{j} \rightarrow 0$ on $\partial \Omega$, the second term converges to zero as $j \rightarrow \infty$. By using Hölder inequality in the first term, we arrive at

$$
\alpha\left\|\nabla u_{j}-\nabla v_{j}\right\|_{L^{2}(\Omega)} \leq \int_{\Omega}\left|\gamma_{j} \nabla u_{j}-F_{j}\right|^{2} d x .
$$


Conclude by (5) and (6). In a similar way, by using the reverse inequality

$$
\int_{\Omega} \beta\left|\nabla u_{j}(x)-\nabla v_{j}(x)\right|^{2} d x \geq \int_{\Omega}\left\langle\gamma_{j}(x)\left(\nabla u_{j}(x)-\nabla v_{j}(x)\right), \nabla u_{j}(x)-\nabla v_{j}(x)\right\rangle d x,
$$

and using the same identities as above, conclude that

$$
\left\|F_{j}-H_{j}\right\|_{L^{2}(\Omega)} \leq \beta\left\|\nabla u_{j}-\nabla v_{j}\right\|_{L^{2}(\Omega)} .
$$

Hence $F_{j}-H_{j}$ converges strongly to zero in $L^{2}$.

It is well-known (see [15]) that the strong convergence just shown implies that the Young measures corresponding to the two sequences of pairs

$$
\left\{\left(F_{j}, \nabla u_{j}\right)\right\}, \quad\left\{\left(H_{j}, \nabla v_{j}\right)\right\}
$$

is in fact the same family of measures. In addition, the sequence of pairs $\left\{\left(H_{j}, \nabla v_{j}\right)\right\}$ can be shown to be equiintegrable in $L^{2}(\Omega)$. This concludes the proof.

As a direct consequence of this proposition, it is clear that our main concern is to deal with probability measures $\nu$ (no dependence on $x$ ) supported in the union $\Lambda_{\alpha} \cup \Lambda_{\beta}$ that are div-curl Young measures. Our strategy is therefore to reformulate the relaxation of the initial optimal design problem in terms of this class of measures.

We turn to the situation of our design problem where $m=1$ so that the state $u$ is a single function, and $\nabla u(x)$ and $F(x)$ are vectors in $\mathbf{R}^{N}$. The relaxation of the original optimal design problem in terms of this family of measures reads as

$$
\text { Minimize in } \nu: \quad \int_{\Omega}\left[a_{\alpha} t(x) \int_{\mathbf{R}^{N}}|\lambda|^{2} d \nu_{x, \alpha}^{(1)}(\lambda)+a_{\beta}(1-t(x)) \int_{\mathbf{R}^{N}}|\lambda|^{2} d \nu_{x, \beta}^{(1)}(\lambda)\right] d x
$$

subject to

$$
\begin{gathered}
\nu=\left\{\nu_{x}\right\}_{x \in \Omega}, \quad \nu_{x}=t(x) \nu_{x, \alpha}+(1-t(x)) \nu_{x, \beta} \text { is a div-curl Young measure, } \\
\operatorname{supp} \nu_{x, \gamma} \subset \Lambda_{\gamma}, \gamma=\alpha, \beta, \quad \int_{\Omega} t(x) d x=t_{0}|\Omega|, \\
F(x)=\int_{\mathbf{R}^{N}} \rho d \nu_{x}(\lambda, \rho), \quad \operatorname{div} F=0 \text { weakly in } \Omega \\
\nabla u(x)=\int_{\mathbf{R}^{N}} \lambda d \nu_{x}(\lambda, \rho), \quad u=u_{0} \text { on } \partial \Omega .
\end{gathered}
$$

To proceed further with the analysis of this relaxed formulation, we regard $x \in \Omega$ as a parameter, and put

$$
\nu=\nu_{x}, \quad \rho=F(x), \quad \lambda=\nabla u(x), \quad t=t(x) .
$$

If we let $\mathrm{CQW}(t, \rho, \lambda)$ stand for the minimum of

$$
a_{\alpha} t \int_{\mathbf{R}^{N}}|x|^{2} d \nu_{\alpha}^{(1)}(x)+a_{\beta}(1-t) \int_{\mathbf{R}^{N}}|x|^{2} d \nu_{\beta}^{(1)}(x)
$$


under the constraints

$$
\begin{gathered}
\nu=t \nu_{\alpha}+(1-t) \nu_{\beta} \text { is a div-curl Young measure, } \quad \operatorname{supp} \nu_{\gamma} \subset \Lambda_{\gamma}, \gamma=\alpha, \beta, \\
\rho=\int_{\mathbf{R}^{N}} y d \nu(x, y), \quad \lambda=\int_{\mathbf{R}^{N}} x d \nu(x, y),
\end{gathered}
$$

then, basically because of the localization property of div-curl Young measures, we find a relaxed formulation at the level of the first moments of div-curl Young measures, namely,

$$
\text { Minimize in }(t, F, u): \quad \int_{\Omega} C Q W(t(x), F(x), \nabla u(x)) d x
$$

subject to

$$
0 \leq t(x) \leq 1, \quad \int_{\Omega} t(x) d x=t_{0}|\Omega|, \quad \operatorname{div} F=0 \text { weakly in } \Omega, \quad u=u_{0} \text { on } \partial \Omega .
$$

See [17] for more details on this passage. The notation CQW comes from the term "constrained quasiconvexification."

It is also of the greatest relevance to detect an optimal feasible measure $\mu_{(t, \rho, \lambda)}$ furnishing the optimal value $\mathrm{CQW}(t, \rho, \lambda)$, because once we find optimal triplets $(t, F, \nabla u)$ for this last variational problem, the family of measures

$$
\nu_{x}=\mu_{(t(x), F(x), \nabla u(x))}
$$

will be optimal for the previous relaxation in terms of measures, and hence will provide the information to build optimal microstructures for our optimal design problem. Our task is to explore and compute $\mathrm{CQW}(t, \rho, \lambda)$, and detect at least one optimal div-curl measure $\mu_{(t, \rho, \lambda)}$.

\section{Relaxation}

Let $\nu$ be a div-curl Young measure supported in the union

$$
\Lambda=\Lambda_{\alpha} \cup \Lambda_{\beta}
$$

where

$$
\Lambda_{\gamma}=\left\{(x, y) \in \mathbf{R}^{N} \times \mathbf{R}^{N}: y=\gamma x\right\},
$$

a linear manifold in $\mathbf{R}^{N} \times \mathbf{R}^{N}$. We can decompose $\nu=t \nu_{\alpha}+(1-t) \nu_{\beta}$ where $\nu_{\gamma}$ is a probability measure (most likely not a div-curl Young measure itself) supported in $\Lambda_{\gamma}$.

Let us look at the first moment of $\nu$

$$
(\lambda, \rho)=\int_{\Lambda}(x, y) d \nu(x, y)=t \int_{\mathbf{R}^{N}}(x, \alpha x) \nu_{\alpha}^{(1)}(x)+(1-t) \int_{\mathbf{R}^{N}}(x, \beta x) \nu_{\beta}^{(1)}(x),
$$


where $\nu_{\gamma}^{(1)}$ is the projection of $\nu_{\gamma}$ onto the first copy of $\mathbf{R}^{N}$ of the product $\mathbf{R}^{N} \times \mathbf{R}^{N}$. If we put

$$
\lambda_{\gamma}=\int_{\mathbf{R}^{N}} x d \nu_{\gamma}^{(1)}(x)
$$

then

$$
\lambda=t \lambda_{\alpha}+(1-t) \lambda_{\beta}, \quad \rho=t \alpha \lambda_{\alpha}+(1-t) \beta \lambda_{\beta} .
$$

From these two identities, we can express $\lambda_{\gamma}$ in terms of $\lambda$ and $\rho$. Namely,

$$
\lambda_{\alpha}=\frac{1}{t(\beta-\alpha)}(\beta \lambda-\rho), \quad \lambda_{\beta}=\frac{1}{(1-t)(\beta-\alpha)}(\rho-\alpha \lambda) .
$$

On the other hand, the commutation with the inner product Lemma 1.3 yields

$$
\int_{\Lambda} x \cdot y d \nu(x, y)=\lambda \cdot \rho
$$

But the integral on the left-hand side can be written

$$
t \alpha \int_{\mathbf{R}^{N}}|x|^{2} d \nu_{\alpha}^{(1)}(x)+(1-t) \beta \int_{\mathbf{R}^{N}}|x|^{2} d \nu_{\beta}^{(1)}(x) .
$$

We will consider the variables

$$
s_{\gamma}=\int_{\mathbf{R}^{N}}|x|^{2} d \nu_{\gamma}^{(1)}(x) \in[0,+\infty) .
$$

By Jensen's inequality we must enforce the constraints

$$
s_{\gamma} \geq\left|\lambda_{\gamma}\right|^{2} .
$$

Let us turn to the cost functional and check that it can be explicitly expressed in terms of these variables $s_{\gamma}$ as well. Indeed, the cost functional is

$$
a_{\alpha} t s_{\alpha}+(1-t) \beta s_{\beta} .
$$

Altogether, we would like to solve the linear programming problem in the variables $\left(s_{\alpha}, s_{\beta}\right)$

$$
\text { Minimize in }\left(s_{\alpha}, s_{\beta}\right) \in \mathbf{R}^{2}: \quad a_{\alpha} t s_{\alpha}+a_{\beta}(1-t) s_{\beta}
$$

subject to the constraints

$$
\begin{gathered}
\lambda \cdot \rho=t \alpha s_{\alpha}+(1-t) \beta s_{\beta}, \\
s_{\alpha} \geq \frac{1}{t^{2}(\beta-\alpha)^{2}}|\beta \lambda-\rho|^{2}, \quad s_{\beta} \geq \frac{1}{(1-t)^{2}(\beta-\alpha)^{2}}|\rho-\alpha \lambda|^{2} .
\end{gathered}
$$


A first issue is to realize that the set of vectors for which the constraints yield a non-empty set takes place if

$$
[\alpha(1-t)+\beta t]|\rho|^{2}+\alpha \beta[\beta(1-t)+\alpha t]|\lambda|^{2}-\left[2 \alpha \beta+t(1-t)(\beta-\alpha)^{2}\right] \lambda \cdot \rho \leq 0 .
$$

This inequality can be rewritten, after some elementary algebra, in a more transparent form as follows

$$
\left|\rho-\frac{2 \alpha \beta+t(1-t)(\beta-\alpha)^{2}}{2[\alpha(1-t)+\beta t]} \lambda\right| \leq \frac{(\beta-\alpha)^{2} t(1-t)}{2[\alpha(1-t)+\beta t]}|\lambda| .
$$

In the variable $\rho$, this condition represents a certain ball in $\mathbf{R}^{N}$ with center and radius depending on $\lambda, t, \alpha$, and $\beta$.

When this relationship between $\lambda$ and $\rho$ holds, then the feasible set for the above linear programming problem is non-empty, in fact it is a bounded segment in $\mathbf{R}^{2}$. Thus, the optimal solution will be attained at one of its two vertices, depending on the particular values of the coefficients $a_{\alpha}$ and $a_{\beta}$. In fact, it is elementary to check, by using the linear relationship between the variables $s_{\alpha}$ and $s_{\beta}$, that if $\alpha a_{\beta}-\beta a_{\alpha} \geq 0$ then the optimal value corresponds to taking

$$
s_{\beta}=\frac{1}{(1-t)^{2}(\beta-\alpha)^{2}}|\rho-\alpha \lambda|^{2},
$$

while if $\alpha a_{\beta}-\beta a_{\alpha} \leq 0$, then the optimal value is found at the vertex with

$$
s_{\alpha}=\frac{1}{t^{2}(\beta-\alpha)^{2}}|\beta \lambda-\rho|^{2} .
$$

It is then easy, but a bit tedious, to perform the computations for any specific choice of these two coefficients.

It is also important to check that all of the feasible triplets $(t, \lambda, \rho)$ are achievable by div-curl laminates. This is again an elementary computation that must be however performed with some care. The version we need here is purely a translation of the same computations in [16]. These can also be found in [17]. Before the precise statement, we introduce some notation. Set

$$
\begin{gathered}
g(\lambda, \rho)=\alpha^{2} \beta^{2}|\lambda|^{4}+|\rho|^{4}+\left(\alpha^{2}+6 \alpha \beta+\beta^{2}\right)(\lambda \cdot \rho)^{2}-2 \alpha \beta|\lambda|^{2}|\rho|^{2} \\
-2(\alpha+\beta)\left(\alpha \beta|\lambda|^{2}+|\rho|^{2}\right) \lambda \cdot \rho, \\
r_{i}=\frac{1}{2}+\frac{1}{2(\beta-\alpha) \lambda \cdot \rho}\left[\alpha \beta|\lambda|^{2}-|\rho|^{2}+(-1)^{i} \sqrt{g(\lambda, \rho)}\right], \quad i=1,2 \\
z=\frac{1}{t(\beta-\alpha)}(\beta \lambda-\rho), \quad w_{i}=\frac{-1}{(\beta-\alpha)\left(1-r_{i}\right)}(\alpha \lambda-\rho), \\
w_{j}=\frac{-r_{j}}{t(\beta-\alpha)\left(1-r_{j}\right)}(\alpha \lambda-\rho), \quad s_{i, j}=\frac{\left(1-r_{i}\right)\left[t\left(1-r_{j}\right)-(1-t) r_{j}\right]}{t\left(1-r_{j}\right)-\left(1-r_{i}\right) r_{j}}, i \neq j .
\end{gathered}
$$


Lemma 3.1. The triplets $(t, \lambda, \rho)$ associated with the vertices of the admissible segment above correspond to second order div-curl laminates in which three pairs of vectors participate. In particular, for the vertex corresponding to

$$
s_{\alpha}=\frac{1}{t^{2}(\beta-\alpha)^{2}}|\beta \lambda-\rho|^{2},
$$

it is given explicitly by

$$
\nu_{i, j}=s_{i, j} \delta_{\left(w_{i}, \beta w_{i}\right)}+\left(1-s_{i, j}\right)\left(\frac{t}{1-s_{i, j}} \delta_{(z, \alpha z)}+\frac{1-s_{i, j}-t}{1-s_{i, j}} \delta_{\left(w_{j}, \beta w_{j}\right)}\right),
$$

for $i \neq j$.

The proof of this lemma is nothing but to check some algebra with the set of formulae given before the statement of the lemma. Namely,

$$
\begin{gathered}
\left(z-w_{j}\right) \cdot\left(\alpha z-\beta w_{j}\right)=0, \\
\left(w_{i}-z_{i, j}\right) \cdot\left(\beta w_{i}-Z_{i, j}\right)=0, \\
z_{i, j}=\frac{t}{1-s_{i, j}} z+\frac{1-s_{i, j}-t}{1-s_{i, j}} w_{j}, \quad Z_{i, j}=\frac{t}{1-s_{i, j}} \alpha z+\frac{1-s_{i, j}-t}{1-s_{i, j}} \beta w_{j}, \\
(\lambda, \rho)=s_{i, j}\left(w_{i}, \beta w_{i}\right)+\left(1-s_{i, j}\right)\left(\frac{t}{1-s_{i, j}}(z, \alpha z)+\frac{1-s_{i, j}-t}{1-s_{i, j}}\left(w_{j}, \beta w_{j}\right)\right) .
\end{gathered}
$$

These div-curl Young measures (and the similar ones for the other vertex) are the optimal measures $\mu_{(t, \rho, \lambda)}$ providing the exact value for $C Q W(t, \rho, \lambda)$. They should be used to build optimal microstructures when we have an optimal solution (or a suitable approximation of it) of the relaxed problem in terms of first moments $(F, \nabla u)$.

We have thus proved that $C Q W(t, \lambda, \rho)$ is the minimum of the preceding linear programming problem, namely the minimum of

$$
\text { Minimize in }\left(s_{\alpha}, s_{\beta}\right) \in \mathbf{R}^{2}: \quad a_{\alpha} t s_{\alpha}+a_{\beta}(1-t) s_{\beta}
$$

subject to the constraints

$$
\begin{gathered}
\lambda \cdot \rho=t \alpha s_{\alpha}+(1-t) \beta s_{\beta}, \\
s_{\alpha} \geq \frac{1}{t^{2}(\beta-\alpha)^{2}}|\beta \lambda-\rho|^{2}, \quad s_{\beta} \geq \frac{1}{(1-t)^{2}(\beta-\alpha)^{2}}|\rho-\alpha \lambda|^{2} .
\end{gathered}
$$

Notice that CQW $=+\infty$ if (8) does not hold. Then, based on the comments at the end of the last section, we can write down an explicit relaxation as follows.

Theorem 3.2. The variational problem

$$
\text { Minimize in }(t, u, F): \quad \int_{\Omega} m(t(x), \nabla u(x), F(x)) d x
$$


subject to

$$
\begin{gathered}
0 \leq t(x) \leq 1, \quad \int_{\Omega} t(x) d x=t_{0}|\Omega|, \\
u \in H^{1}(\Omega), u=u_{0} \text { on } \partial \Omega, \quad F \in L^{2}(\Omega), \operatorname{div} F=0 \text { weakly in } \Omega,
\end{gathered}
$$

is a relaxation of the initial optimal design problem in the sense that it admits optimal solutions, its minimum coincides with the infimum of the original problem, and optimal solutions encode, in the sense of Young measures, the optimal microstructures for the design problem.

As an illustration, take $a_{\beta}=1, a_{\alpha}=0$. Then the optimal solution in the linear programming problem defining CQW is achieved for

$$
s_{\beta}=\frac{1}{(1-t)^{2}(\beta-\alpha)^{2}}|\rho-\alpha \lambda|^{2},
$$

and the value of the minimum is

$$
\operatorname{CQW}(t, \lambda, \rho)=\frac{1}{(1-t)(\beta-\alpha)^{2}}|\rho-\alpha \lambda|^{2}
$$

if (8) holds. If not, then the infimum is $+\infty$ as the feasible set for the mathematical programming problem is empty. For this particular choice of the coefficients $a_{\alpha}, a_{\beta}$, we therefore have

$$
\operatorname{CQW}(t, \lambda, \rho)= \begin{cases}\frac{1}{(1-t)(\beta-\alpha)^{2}}|\rho-\alpha \lambda|^{2}, & \text { if (8) holds, } \\ +\infty, & \text { else. }\end{cases}
$$

\section{A final transformation for the relaxed problem}

The form of the relaxed problem in Theorem 3.2 is somewhat inappropriate, as it does not show a similar structure as the optimal design problem it comes from. The main drawback is that we do not have a state equation as such. We now elaborate a bit that relaxation in order to write it in a more familiar form.

We introduce an additional design variable $s \in B$ where $B$ is the unit ball in $\mathbf{R}^{N}$. The relationship between $t, s, \lambda$, and $\rho$ comes from (8). Indeed, if we put

$$
s=\frac{2[\alpha(1-t)+\beta t]}{t(1-t)(\beta-\alpha)^{2}|\lambda|} \rho-\frac{2 \alpha \beta+t(1-t)(\beta-\alpha)^{2}}{t(1-t)(\beta-\alpha)^{2}} \frac{\lambda}{|\lambda|},
$$

then $s \in B$. So that

$$
\rho=\frac{2 \alpha \beta+t(1-t)(\beta-\alpha)^{2}}{2[\alpha(1-t)+\beta t]} \lambda+\frac{t(1-t)(\beta-\alpha)^{2}}{2[\alpha(1-t)+\beta t]}|\lambda| s .
$$


Put even further

$$
\varphi(t, s, \lambda)=\frac{2 \alpha \beta+t(1-t)(\beta-\alpha)^{2}}{2[\alpha(1-t)+\beta t]} \lambda+\frac{t(1-t)(\beta-\alpha)^{2}}{2[\alpha(1-t)+\beta t]}|\lambda| s .
$$

Since $\rho$ is the variable for $F$ in Theorem 3.2, we are led to consider the state equation

$$
\operatorname{div} \varphi(t(x), s(x), \nabla u(x))=0 \text { in } \Omega, \quad u=u_{0} \text { on } \partial \Omega .
$$

This is a non-linear, regular elliptic equation because it comes from a relaxation of a well-posed elliptic problem (for each fixed $\chi$ ). After some elementary algebra, the cost density can be written in the particular case $a_{\beta}=1, a_{\alpha}=0$, as

$$
\psi(t, s, \lambda)=\frac{(1-t)}{4[\alpha(1-t)+\beta t]^{2}}|(2 \alpha+t(\beta-\alpha)) \lambda+t(\beta-\alpha)| \lambda|s|^{2} .
$$

In general $\psi$ and CQW are related through (9) and (10). Therefore, our final relaxed problem, through which we can understand optimal microstructures for our initial optimal design problem, is precisely the one stated in Theorem 0.1. Once we have solved this new relaxed optimal design problem in the form $(t(x), s(x), \nabla u(x))$, optimal div-curl laminates are found by first using (10) to find $F(x)(=\rho(x))$, and then by putting

$$
\nu_{x}=\mu_{(t(x), F(x), \nabla u(x))}
$$

according to (7) and Lemma 3.1.

\section{References}

[1] G. Allaire, Shape optimization by the homogenization method, Applied Mathematical Sciences, vol. 146, Springer-Verlag, New York, 2002.

[2] E. Aranda and P. Pedregal, Constrained envelope for a general class of design problems, Dynamical Systems and Differential Equations (Wilmington, NC, 2002), 2003, pp. 30-41.

[3] E. J. Balder, Lectures on Young Measures, Cahiers de Mathématiques de la Décision, CEREMADE, Université Paris IX, 1995.

[4] J. C. Bellido, Explicit computation of the relaxed density coming from a three-dimensional optimal design problem, Nonlinear Anal. 52 (2003), no. 7, 1709-1726.

[5] M. P. Bendsøe, Optimization of structural topology, shape, and material, Springer-Verlag, Berlin, 1995.

[6] I. Fonseca and S. Müller, A-quasiconvexity, lower semicontinuity, and Young measures, SIAM J. Math. Anal. 30 (1999), no. 6, 1355-1390.

[7] P. R. Kotiuga, Clebsch potentials and the visualization of three-dimensional solenoidal vector fields, IEEE Trans. Magnetics 27 (1991), no. 5, 3986-3989.

[8] F. Maestre and P. Pedregal, Quasiconvexification in 3-D for a variational reformulation of an optimal design problem in conductivity, Nonlinear Anal. 64 (2006), no. 9, 1962-1976.

[9] S. Müller, Variational models for microstructure and phase transitions, Calculus of Variations and Geometric Evolution Problems (Cetraro, 1996), Lecture Notes in Math., vol. 1713, Springer, Berlin, 1999, pp. 85-210. 
[10] F. Murat, A survey on compensated compactness, Contributions to Modern Calculus of Variations (Bologna, 1985) (L. Cesari, ed.), Pitman Res. Notes Math. Ser., vol. 148, Longman Sci. Tech., Harlow, 1987, pp. 145-183.

[11] F. Murat and L. Tartar, On the control of coefficients in partial differential equations, Topics in the mathematical modelling of composite materials (A. Cherkaev and R. V. Kohn, eds.), Progr. Nonlinear Differential Equations Appl., vol. 31, Birkhäuser, Boston, MA, 1997, pp. 1-8.

[12] _ Calcul des variations et homogénéisation, Les méthodes de l'homogénéisation: théorie et applications en physique (Bréau-sans-Nappe, 1983), Collect. Dir. Études Rech. Élec. France, vol. 57, Eyrolles, Paris, 1985, pp. 319-369.

[13] _ Optimality conditions and homogenization, Nonlinear Variational Problems (Isola d'Elba, 1983), Res. Notes in Math., vol. 127, Pitman, Boston, MA, 1985, pp. 1-8.

[14] S. D. Nigam, R. Usha, and K. Swaminathan, Divergence-free vector fields, J. Math. Phys. Sci. 14 (1980), no. 5, 523-527.

[15] P. Pedregal, Parametrized measures and variational principles, Progress in Nonlinear Differential Equations and Their Applications, vol. 30, Birkhäuser Verlag, Basel, 1997.

[16] Constrained quasiconvexification of the square of the gradient of the state in optimal design, Quart. Appl. Math. 62 (2004), no. 3, 459-470.

[17] _ Vector variational problems and applications to optimal design, ESAIM Control Optim. Calc. Var. 11 (2005), no. 3, 357-381.

[18] P. M. Santos, $\mathcal{A}$-quasi-convexity with variable coefficients, Proc. Roy. Soc. Edinburgh Sect. A 134 (2004), no. 6, 1219-1237.

[19] L. Tartar, Compensated compactness and applications to partial differential equations, Nonlinear analysis and mechanics: Heriot-Watt Symposium, vol. IV (R. Knops, ed.), Res. Notes in Math., vol. 39, Pitman, Boston, Mass., 1979, pp. 136-212.

[20] _ Remarks on optimal design problems, Calculus of Variations, Homogenization and Continuum Mechanics (Marseille, 1993) (G. Buttazzo, G. Bouchitte, and P. Suquet, eds.), Ser. Adv. Math. Appl. Sci., vol. 18, World Sci. Publ., River Edge, NJ, 1994, pp. 279-296.

[21] __ An introduction to the homogenization method in optimal design, Optimal Shape Design (Tróia, 1998), Lecture Notes in Math., vol. 1740, Springer, Berlin, 2000, pp. 47-156.

[22] M. Valadier, Young measures, Methods of Nonconvex Analysis (Varenna, 1989), Lecture Notes in Math., vol. 1446, Springer, Berlin, 1990, pp. 152-188.

[23] C.-S. Yih, Stream functions in three-dimensional flows, Houille Blanche 12 (1957), 445-450.

[24] L. C. Young, Generalized surfaces in the calculus of variations. I, 84-103; II, 530-544, Ann. of Math. (2) 43 (1942). 